\title{
POLYNOMIAL HULLS WITH CONVEX SECTIONS AND INTERPOLATING SPACES
}

\author{
ZBIGNIEW SLODKOWSKI
}

\begin{abstract}
Assume that $L \subset \partial D \times \mathbf{C}^{m}$ is compact and has convex vertical sections. Denote by $K$ its polynomially convex hull. It is shown that $K \backslash \partial D \times \mathbf{C}^{m}$, if nonempty, can be covered by graphs of analytic functions $f: D \rightarrow \mathbf{C}^{m}$. The proof is based on complex interpolation theory for families of finite-dimensional normed spaces.
\end{abstract}

1. Introduction. If $L$ is a subset of $\mathbf{C}^{m+1}$ and $z \in \mathbf{C}$, denote

$$
L(z)=\left\{w \in \mathbf{C}^{m}:(z, w) \in L\right\}
$$

we will refer to $L(z)$ as a section or fiber over $z$. In what follows $D$ denotes the open unit disc.

The purpose of this paper is to present a proof of the following theorem.

THEOREM 1. Let $L$ be a compact subset of $\partial D \times \mathbf{C}^{m}$. Assume that for every $z \in \partial D$ the section $L(z)$ is a nonempty compact and convex subset of $\mathbf{C}^{m}$. Denote by $K$ the polynomially convex hull of $L$. Then for every $\left(z^{*}, w^{*}\right)$ in $K$ such that $z^{*} \in D$, there exists a bounded analytic function $f: D \rightarrow \mathbf{C}^{m}$ such that $f\left(z^{*}\right)=w^{*}$ and $f(z) \in K(z)$ for every $z \in D$.

Two different proofs of this theorem, which generalizes the earlier approximation result of Alexander and Wermer [1], were obtained independently by $\mathrm{H}$. Alexander and J. Wermer on the one hand, and by the author on the other.

The proof given below is based on the theory of complex interpolation for families of finite-dimensional normed spaces as presented in Coifman et al. [4] and, at least for this reason, is not simpler than that of Alexander and Wermer. However, our proof is perhaps not without interest since it is carried over for arbitrary dimension $m$ (the paper of Alexander and Wermer is focused on the case $m=1$ ) and presents a new instance of relationship between the theory of complex interpolation of Banach spaces and the theory of analytic multifunctions. Namely, if $K \neq L$, then the multifunction $z \rightarrow K(z): D \rightarrow 2^{\mathrm{C}^{m}}$ is analytic in the sense of [7]. A different type of relationship between the two theories was described in [8].

For more information on analytic multifunctions the reader is referred to $[3,5,6]$.

Received by the editors February 15, 1985.

1980 Mathematics Subject Classification. Primary 32E20; Secondary 46M35.

(C)1986 American Mathematical Society $0002-9939 / 86 \$ 1.00+\$ .25$ per page 


\section{Method of proof.}

Notation. The polynomially convex hull of a set $X \subset \mathbf{C}^{m}$ is denoted by hull $(X)$ and convex hull by $\operatorname{co}(X)$. The usual bilinear product $\sum v_{i} z_{i}$ in $\mathbf{C}^{n}$ is denoted by $\langle v, z\rangle$, and if $X$ is a subset of $\mathbf{C}^{n}$, then the set

$$
X^{0}=\left\{v \in \mathbf{C}^{n}:|\langle v, z\rangle| \leqslant 1 \text {, for } z \in X\right\}
$$

denotes the (closed) polar of $X$. Subsets of $\mathbf{C}^{m+1}$, e.g. $L \subset \partial D \times \mathbf{C}^{m}$, will be looked upon as graphs of set-valued functions (= multifunctions), e.g. $z \rightarrow L(z): \partial D \rightarrow 2^{\mathrm{C}^{m}}$ (in case the projection of $L$ on $\mathbf{C}^{1}$ fills $\partial D$ ).

The proof of Theorem 1 will be easily reduced to the case of sets $L$ for which the multifunction $z \rightarrow L(z): \partial D \rightarrow 2^{\mathbf{C}^{m}}$ is continuous. Assuming this we fix $r \in(0,1)$ and for each $z \in \partial D$ define the set $B(z) \subset \mathbf{C}^{m+1}$ as the convex hull of the union of the two sets

$$
\begin{gathered}
\left\{(w, u) \in \mathbf{C}^{m} \times \mathbf{C}:|w|^{2}+|u|^{2} \leqslant r\right\}, \\
\left\{(w u, u) \in \mathbf{C}^{m} \times \mathbf{C}: w \in L(z),|u| \leqslant 1\right\} .
\end{gathered}
$$

Proposition 2. If $z \rightarrow L(z): \partial D \rightarrow 2^{\mathbf{C}^{m}}$ is a continuous multifunction with all $L(z)$ convex and compact then the multifunction $z \rightarrow B(z): \partial D \rightarrow 2^{\mathrm{C}^{m+1}}$, defined as above, is continuous and all $B(z)$ are norming bodies ( = convex circled and compact sets with nonempty interior).

Since every $B(z), z \in \partial D$, defines a norm on $\mathbf{C}^{m+1}$, the complex interpolation method of Coifman et al. [4] yields intermediate norms for all $z$ in $D$. We find it more convenient to consider the family of closed norming bodies $\{W(z): z \in D\}$ corresponding to these norms. We summarize now and reformulate in terms of $\{W(z)\}$ those results of [4] which we use below. (Note the weakened form of these results.)

Theorem 3 (CoIfMAN eT AL. [4], Theorems I AND II). If

$$
z \rightarrow B(z): \partial D \rightarrow 2^{C^{M}}
$$

is a continuous multifunction whose values are compact norming bodies, then there exists a family of compact norming bodies

$$
z \rightarrow W(z): \partial D \rightarrow 2^{\mathrm{C}^{M}}
$$

such that $W\left(e^{i \theta}\right)=B\left(e^{i \theta}\right)$ and

(i) for every $a \in D$ and $b \in W(a)$ there exists a bounded analytic function $f$ : $D \rightarrow 2^{\mathbf{C}^{M}}$ such that $f(a)=b, f(z) \in W(z)$ for every $z \in D$ and $\tilde{f}\left(e^{i \theta}\right) \in B\left(e^{i \theta}\right)$ a.e. $(\tilde{f}(\cdot)$ denotes nontangential boundary value $)$;

(ii) the same condition as (i) with sets $B\left(e^{i \theta}\right)$ and $W(z)$ replaced by their closed polars $B\left(e^{i \theta}\right)^{\circ}$ and $W(z)^{\circ}(c f .(1))$ holds.

Using these facts we can reinterpret family $W(\cdot)$ in terms of hulls, which is the first step of our proof. 
THEOREM 4. Under the assumptions of Theorem $3 W=\operatorname{hull}(B)$, where

$$
W=\{(z, w): z \in \bar{D}, w \in W(z)\} ; \quad B=\{(z, w): z \in \partial D, w \in B(z)\} .
$$

The essence of our argument is to apply this description to multifunction $B(\cdot)$ obtained from $L(\cdot)$ by (2), (3). It follows that $W \cap\{u=1\}=K \times\{1\}$ and so the latter set is, in a sense, a face of $W$. Therefore those graphs of analytic functions $f$ : $D \rightarrow 2^{\mathrm{C}^{m+1}}$ which pass through points of $K \times\{1\}$ and are contained in $W$ have to be contained in $K \times\{1\}$ as well. By Theorem 4 such graphs are in abundance and so $K \times\{1\}$ is covered by them. This settles Theorem 1 in case multifunction $L(\cdot)$ is continuous.

3. Proof of Theorem 4. We consider first three simple lemmas to get continuity of $W(\cdot)$ under assumption of continuity of $B(\cdot)$.

LEMMA 5. Under the assumptions of Theorem 3,

(i) $\operatorname{hull}(B) \cap \partial D \times C^{M}=B$,

(ii) $W \subset \operatorname{hull}(B)$.

Proof. (i) We will show that if $a \in \partial D$ and $b \in \mathbf{C}^{M} \backslash B(a)$ then $(a, b) \notin \operatorname{hull}(B)$. Take $r>\mathbf{1}$ such that $(b / r) \in \partial B(a)$ and a $\mathbf{C}$-linear form $l: \mathbf{C}^{M} \rightarrow \mathbf{C}$ such that $l(b)=r$ and $|l(w)| \leqslant 1$ for $w \in B(a)$. Set

$$
p_{k}(z, w)=((1+z a) / 2)^{k} l(w),
$$

where $k$ is an integer. Note that the sequence $\left|p_{k}(z, w)\right|$ is nonincreasing on $B$ and converges pointwise on $B$ to the (upper semicontinuous) characteristic function of the set $\{(z, w) \in B: \quad z=a, l(w)=1\}$. Thus (cf. [6, Lemma 2.3, Assertion]) $\lim _{k}\left(\max _{B}\left|p_{k}\right|\right)=1$, and so $\max _{B}\left|p_{k}\right|$ is smaller than $p_{k}(a, b)=r$ for $k$ large enough, i.e., $(a, b) \notin \operatorname{hull}(B)$.

(ii) Let $a \in D, b \in W(a)$ and $p(z, w)$ be a complex polynomial such that $\max _{B}|p| \leqslant 1$; we have to show that $|p(a, b)| \leqslant 1$. Let $f$ be a function satisfying literally condition (i) of Theorem 3. Set $h(z)=p(z, f(z)), z \in D$. Of course $h$ is a bounded holomorphic function whose nontangential boundary values are $\tilde{h}\left(e^{i \theta}\right)=$ $p\left(e^{i \theta}, \tilde{f}\left(e^{i \theta}\right)\right)$ a.e. Since $|p| \leqslant 1$ on $B$ and $\tilde{f}\left(e^{i \theta}\right) \in B\left(e^{i \theta}\right)$ a.e., therefore $\|h\|_{\infty} \leqslant 1$ and so $|p(a, b)|=|h(a)| \leqslant 1$. Q.E.D.

We omit the simple topological proof of the next lemma.

LEMMA 6. Let $(S, d)$ be a compact metric space and let $B(z)$ be a compact norming body in $\mathbf{C}^{M}$ for every $z \in S$. Then the following two conditions are equivalent:

(i) multifunction $z \rightarrow B(z): S \rightarrow 2^{C^{M}}$ is continuous;

(ii) for every $\varepsilon>0$ there is $\delta>0$, such that whenever $z^{\prime}, z^{\prime \prime} \in S$ and $d\left(z^{\prime}, z^{\prime \prime}\right)<\delta$, then $B\left(z^{\prime}\right) \subset(1+\varepsilon) B\left(z^{\prime \prime}\right)$.

LEMMA 7. Under the assumptions of Theorem 3 the multifunction

$$
z \rightarrow W(z): \bar{D} \rightarrow 2^{\mathrm{C}^{M}}
$$

is continuous.

Proof. $1^{\circ}$. Continuity at points of the open disc. The lower semicontinuity of $W(\cdot)$ at $z=a,|a|<1$, follows immediately from condition (i) of Theorem 3 . 
To prove upper semicontinuity at $z=a \in D$, consider sequences $a_{k} \rightarrow a$ and $b_{k} \rightarrow b$ such that $b_{k} \in W\left(a_{k}\right)$; we have to show that $b \in W(a)$. Choose by Theorem 3(i) analytic functions $f_{k}: D \rightarrow \mathbf{C}^{M}$, such that

$$
f_{k}\left(a_{k}\right)=b_{k} ; \quad f_{k}(z) \in W(z), \quad k=1,2, \ldots, z \in D .
$$

Select by Montel's theorem a subsequence $f_{k(n)}$ converging uniformly on compact subsets of $D$ to a function $f$. The sequence $\left\{a_{k}\right\}$ being relatively compact in $D$,

$$
f(a)=\lim _{n} f_{k(n)}\left(a_{k(n)}\right)=b .
$$

On the other hand $f(a)=\lim _{n} f_{k(n)}(a) \in W(a)$ by (5) and so $b \in W(a)$, as required.

$2^{\circ}$. Continuity at boundary points. Let $a \in \partial D, a_{k} \in D, a_{k} \rightarrow a, b_{k} \in W\left(a_{k}\right)$ and $b_{k} \rightarrow b$. By Lemma 5(ii), $\left(a_{k}, b_{k}\right) \in \operatorname{hull}(B)$ and so $(a, b) \in \operatorname{hull}(B)$. By Lemma 5(i) $(a, b) \in B$, i.e., $b \in B(a)=W(a)$, which proves upper semicontinuity of $W(\cdot)$ at $z=a$.

Define now for $a \in \partial D, b \in \mathbf{C}^{M}$ and for an integer $k$, the polynomial mapping $p_{k}(z)=((1+z \bar{a}) / 2)^{k} b$. Note that $p_{k}(a)=b$.

In order to confirm that $W(\cdot)$ is lower semicontinuous at $z=a$ it suffices to show that for every $b$ in $\operatorname{Int} W(a)$ there exists an integer $k$ such that $p_{k}(z) \in W(z)$ for every $z \in \bar{D}$. Observe first that by Lemma 6(ii) and the fact that $\left|p_{k}(z)\right| \searrow 0$ on $\partial D \backslash\{a\}$ we can choose $k$ such that

$$
p\left(e^{i \theta}\right) \in W\left(e^{i \theta}\right), \quad e^{i \theta} \in \partial D .
$$

Accepting this, take $z^{\prime} \in D$ and $v \in W\left(z^{\prime}\right)^{\circ}$, and apply Theorem 3(ii) to get a bounded analytic function $g: D \rightarrow \mathbf{C}^{M}$ such that $g\left(z^{\prime}\right)=v$ and $\tilde{g}\left(e^{i \theta}\right) \in B\left(e^{i \theta}\right)^{\circ}$ a.e. By $(6)\left|\left\langle\tilde{g}\left(e^{i \theta}\right), p_{k}\left(e^{i \theta}\right)\right\rangle\right| \leqslant 1$ a.e. and therefore

$$
\left|\left\langle v, p_{k}\left(z^{\prime}\right)\right\rangle\right|=\left|\left\langle g\left(z^{\prime}\right), p_{k}\left(z^{\prime}\right)\right\rangle\right| \leqslant \sup \operatorname{ess}\left|\left\langle\tilde{g}\left(e^{i \theta}\right), p_{k}\left(e^{i \theta}\right)\right\rangle\right| \leqslant 1 .
$$

Vector $v$ being arbitrary in $W\left(z^{\prime}\right)^{\circ}$, we conclude that $p_{k}\left(z^{\prime}\right) \in W\left(z^{\prime}\right)$, which ends the proof of lower semicontinuity of $W(\cdot)$. Q.E.D.

Proof of Theorem 4. Since by Lemma 5(ii)

$$
W \subset \operatorname{hull}(B) \subset \operatorname{hull}(W),
$$

it suffices to show that $W$ is polynomially convex. We need the following Assertion.

ASSERTION. For every $R \in(0,1)$ the set

$$
Y=\{(z, w):|z| \leqslant R, w \in W(z)\}
$$

is polynomially convex.

To check this, fix $a \in D$ with $|a| \leqslant R$ and $b \in \mathbf{C}^{M} \backslash W(a)$; we will find a polynomial $p(z, w)$ such that $|p(a, b)|$ is greater than $\max _{Y}|p|$. Let $\alpha>1$ be such that $(b / \alpha) \in \partial B(a)$ and choose $v \in B(a)^{\circ}$ such that $\langle v, b\rangle=\alpha$. Choose by Theorem 3(ii) an analytic function $g: D \rightarrow \mathbf{C}^{m}$ such that $g(z) \in B(z)^{\circ}$ for $z \in D$. Set $h(z, w)=\langle g(z), w\rangle$ on $D \times \mathbf{C}^{M}$. Then $h$ is an analytic function such that $\alpha=$ $h(a, b)>\max _{Y}|h|=1$. Choose a compact polydisc contained in $D \times \mathbf{C}^{M}$ and containing $Y \cup\{(a, b)\}$; approximating $h$ by polynomials uniformly on this polydisc we find a polynomial $p$ such that $|p(a, b)|$ is greater than $\max _{Y}|p|$, which proves the Assertion. 
By Lemma 6(ii) for every $k=2,3,4, \ldots$, there exists $r_{k} \in\left(1-k^{-1}, 1\right)$ such that for every $z \in \bar{D}$

$$
W(z) \subset\left(1+k^{-1}\right) W\left(r_{k} z\right) \subset\left(1+k^{-1}\right)^{2} W(z) .
$$

Define

$$
Y_{k}=\left\{(z, w): z \in \bar{D}, w \in\left(1+k^{-1}\right) W\left(r_{k} z\right)\right\} \text {. }
$$

By inclusions (8) $W(z)=\bigcap_{k}\left(1+k^{-1}\right) W\left(r_{k} z\right)$ for $z \in \bar{D}$ and so $W=\bigcap_{k} Y_{k}$. Since each $Y_{k}$ is the image, by a nonsingular linear transformation, of the set (7) with $R=r_{k}$, each $Y_{k}$ is polynomially convex and so is $W$. Q.E.D.

\section{Proof of Theorem 1.}

Proof of Proposition 2. The compactness of $B(z)$ follows from a theorem due to Carathéodory by which each point in the convex hull of a subset $X$ of $R^{N}$ is a convex combination of $N+1$ points of $X$. The same theorem yields easily the proof that the multifunction $B(\cdot)$ is both lower and upper semicontinuous provided $L(\cdot)$ is.

Lemma 8. Let $L$ and $K$ be as in Theorem 1. Assume in addition that the multifunction $z \rightarrow L(z)$ is continuous, and let $W$ be defined as in $\S 2$. Then

(i) $K \times\{1\}=W \cap\{u=1\}$,

(ii) the statement of Theorem 1 holds.

Proof. (i) By definition of $B(z)$ (cf. (2), (3)) $B(z) \cap\{u=1\}=L(z) \times\{1\}$ for $z \in \partial D$ and so

$$
B \cap\{u=1\}=L \times\{1\} .
$$

Set $Z=W \cap\{u=1\}$. By Theorem 4 the graph $W$ is polynomially convex and so is $Z$. Since

therefore

$$
Z \cap\left(\partial D \times C^{m+1}\right)=B \cap\{u=1\}=L \times\{1\}
$$

$$
K \times\{1\}=\operatorname{hull}(L) \times\{1\}=\operatorname{hull}(L \times\{1\}) \subset Z .
$$

Suppose that the reverse inclusion does not hold, i.e., there exists a polynomial $p(z, w, u)$ such that

$$
\max _{Z}|p|=1>\max _{L \times\{1\}}|p|
$$

In this case consider polynomials

$$
q_{k}(z, w, u)=p(z, w, u)((1 / 2)(1+u))^{k} .
$$

By (10) and the inclusion $B \subset Z \cup\{|(1 / 2)(1+u)|<1\}$ there exists $k$ such that $\max _{W}\left|q_{k}\right|=1>\max _{B}\left|q_{k}\right|$, which contradicts Theorem 4. Part (i) is proved.

(ii) Let $a \in D$ and $b \in K(a)$. By part (i) $(b, 1) \in W(a)$ and by Theorem 3(i) there exists a bounded analytic function $(f, g): D \rightarrow \mathbf{C}^{m} \times \mathbf{C}$ such that $f(a)=b, g(a)=$ 1 and $(f(z), g(z)) \in W(z)$ for $z \in D$. Since $W(z) \subset\{|u| \leqslant 1\},\|g\|_{\infty} \leqslant 1$ and so $g \equiv 1$ in $D$, that is $(f(z), 1) \in W(z)$ for $z \in D$. By part (i) $f(z) \in K(z)$ for all $z \in D$. Q.E.D.

The proof of the next proposition will be given after the proof of Theorem 1 . 
Proposition 9. Let $L \subset \partial D \times \mathbf{R}^{N}$ be a compact set such that for every $z \in \partial D$ the section $L(z)$ is a nonempty compact convex set. Then there exists a decreasing sequence of compact subsets of $\partial D \times \mathbf{R}^{N}$ whose intersection is equal to $L$ and such that the multifunctions

$$
z \rightarrow L_{n}(z): \partial D \rightarrow 2^{\mathbf{R}^{N}}, \quad n=1,2, \ldots
$$

are continuous and all sections $L_{n}(z)$ are convex and compact.

Proof of Theorem 1. Let set $L \subset \partial D \times \mathbf{C}^{m}$ be as in Theorem 1 and let $L_{n}$ be a sequence of subsets of $\partial D \times \mathbf{C}^{m}$ satisfying conditions of Proposition 9. Let $K_{n}=$ $\operatorname{hull}\left(L_{n}\right), n=1,2, \ldots$. Since $\bigcap_{n} \operatorname{hull}\left(L_{n}\right)=\operatorname{hull}(L)$ (note $\left.L_{n} \supset L_{n+1}\right)$, therefore $K=\bigcap_{n} K_{n}$. If $a \in D$ and $b \in K(a)$, then $b \in K_{n}(a)$ and applying Lemma 7(ii) one obtains a sequence of analytic functions $f_{n}: D \rightarrow \mathbf{C}^{m}$ such that $f_{n}(a)=b$ and $f_{n}(z) \in K_{n}(z)$ for $z \in D$. This sequence, being uniformly bounded, has a subsequence convergent to a function $f$ such that $f(a)=b$ and $f(z) \in K(z)$ for $z \in D$. Q.E.D.

Proof of Proposition 9 (Sketch). It is more convenient to consider $L \subset[0,2 \pi]$ $\times \mathbf{R}^{N}$ with $L(0)=L(2 \pi)$; we will obtain approximations $L_{n}$ such that $L_{n}(0)=$ $L_{n}(2 \pi)$. Let $0=t_{0}<t_{1}<\cdots<t_{n}=2 \pi$ and let $S_{i}=\operatorname{co\bigcup }\left\{L(t): t_{i-1} \leqslant t \leqslant\right.$ $\left.\left.t_{i+1}\right\}\right)$. Then $S_{i} \cap S_{i+1}$ contains $L(t)$ for $t_{i}<t<t_{i+1}$. Define $L^{*}$ as the union of all segments with endpoints on $\left\{t_{i}\right\} \times S_{i}$ and $\left\{t_{i+1}\right\} \times S_{i+1}, 0 \leqslant i \leqslant n-1$. It is clear that $L^{*}$ contains $L$ and the multifunction $t \rightarrow L^{*}(t)$ is continuous. (Also $L^{*}(0)=$ $L^{*}(2 \pi)$.) Set $L_{n}, n=1,2, \ldots$, as $L^{*}$ corresponding to the $n$th diadic division. The sequence $L_{n}$ fulfills our claim. (We omit further details.)

Acknowledgement. The author is grateful to Professor John Wermer for his interest in the results of this paper.

\section{REFERENCES}

1. H. Alexander and J. Wermer, On the approximation of singularity sets by analytic varieties, Pacific J. Math. 104 (1983), 263-268.

2. __ Polynomial hulls with convex fibers, Math. Ann. 27 (1985), 99-109.

3. B. Aupetit, Analytic multivalued functions in Banach algebras, Adv. in Math. 44 (1982), 18-60.

4. R. Coifman, M. Gwikel, R. Rochberg, Y. Sagher and G. Weiss, The complex method for interpolation of operators acting on families of Banach spaces, Lecture Notes in Math., Vol. 779, Springer-Verlag, Berlin and New York, 1980, pp. 123-153.

5. T. J. Ransford, Analytic multivalued functions, Ph.D. Thesis, University of Cambridge, 1983.

6. Z. Slodkowski, Analytic set-valued functions and spectra, Math. Ann. 256 (1981), 363-386.

7. Analytic multifunctions, q-plurisubharmonic functions and uniform algebras (Proc. Conf. Banach algebras and several complex variables), F. Greenleaf and D. Gulick, editors, Contemp. Math., vol. 32, Amer. Math. Soc., Providence, R. I., 1984, pp. 243-258.

8. , A generalization of Vesentini and Wermer's theorems. Rend. Sem. Mat. Univ. Padova (to appear).

Department of Mathematics, University of California, los Angeles, California 90024

Current address: Department of Mathematics, Statistics and Computer Science, The University of Illinois at Chicago, Box 4348, Chicago, Illinois 60680 